UMN-TH-1423/96

TPI-MINN-96/2

February 1996

\title{
Electric Dipole Moment Constraints on Phases in the Constrained MSSM
}

\author{
Toby Falk and Keith A. Olive \\ School of Physics and Astronomy, University of Minnesota, Minneapolis, MN 55455, USA
}

\begin{abstract}
We consider constraints on $C P$-violating phases in the Constrained Minimal Supersymmetric Standard Model. We find that by combining cosmological limits on gaugino masses with experimental bounds on the neutron and electron electric dipole moments, we can constrain the phase of the Higgs mixing mass $\mu$ to be $\left|\theta_{\mu}\right|<\pi / 10$, independent of choices of the other mass parameters in the model. The other $C P$-violating phase $\theta_{A}$ is essentially unconstrained.
\end{abstract}


The difficulties associated with trying to constrain the vast parameter space of the general low-energy Minimal Supersymmetric Standard model are well known, and some simplifying assumptions are usually be made in order to get an experimental handle on the set of new parameters. A common ansätz is that of the Constrained Minimal Supersymmetric Standard Model, based on supergravity and grand unification (described in more detail below). The CMSSM is interesting because it is simple, predictive and naturally provides a stable dark matter candidate [1] (an LSP bino-type neutralino) over most of its parameter space. In [2], we showed that there was a strong correlation between the $C P$-violating phases in the MSSM, the cosmological LSP relic density and the neutron and electron electric dipole moments, when all of the parameters are set at the weak scale and hence are independent of any RGE evolution. Here we wish to consider the effect of the $C P$-violating phases present (though normally ignored) in the CMSSM, specifically the inducing of electric dipole moments for the neutron and the electron. We will find that by combining cosmological constraints on the mass of the LSP with experimental bounds on the neutron and electron EDM's, we can bound one of the two new phases in the CMSSM to lie within $\left|\theta_{\mu}\right|<\pi / 10$.

The MSSM contains a plethora of new parameters, which make empirically testing the model difficult. In addition to the supersymmetric Higgs mixing mass parameter $\mu$, there are supersymmetry-breaking gaugino masses $M_{i}$, sfermion mass ${ }^{2}$ parameters $m_{\tilde{f}_{i}}^{2}$, trilinear scalar parameters $A_{i}$, the Higgs scalar mixing mass ${ }^{2} B \mu$, and the ratio of the Higgs vevs, $\tan \beta$. This large parameter space can be simplified at the unification scale by taking a common gaugino mass $M$, sfermion mass ${ }^{2}$ parameter $m_{0}^{2}$ and trilinear scalar parameter $A$, and this model is referred to as the CMSSM. Since in the CMSSM, the two Higgs mass ${ }^{2}$ parameters are set equal to the sfermion mass ${ }^{2}$ parameters at $M_{X}$, the other Higgs sector masses are determined by the requirement of correct electroweak symmetry breaking, once $\tan \beta$ and $m_{\text {top }}$ are fixed. In principle, $M, \mu, B \mu$, and $A$ may be complex; however, not all of these phases are physical [3]. It is possible to rotate away the phase of the gaugino masses. And, by making $B \mu$ real, we ensure that the vacuum expectation values of the Higgs fields are real. The CMSSM, then, is specified by three masses $\left(m_{0}, M\right.$, and $\left.A_{0}\right)$, two phases $\left(\theta_{\mu}\left(M_{X}\right)\right.$ and $\left.\theta_{A}\left(M_{X}\right)\right)$, $\tan \beta$ and $m_{\text {top }}$.

Once the masses and phases are given at $M_{X}$, they can be RGE evolved to the electroweak scale. In practice, we use the one-loop RGEs for the masses and two-loop RGEs for the gauge and Yukawa couplings [4]. The structure of the equations for the gauge couplings, gaugino masses and the diagonal elements of the sfermion masses are such that they are entirely real. The evolutions of the $A_{i}$, however, are more complicated, as the $A_{i}$ pick up both real and 
imaginary contributions. For example, the evolution of $A_{t}$ is given by

$$
\frac{d A_{t}}{d t}=\frac{1}{8 \pi^{2}}\left(-\frac{16}{3} g_{3}^{2} M_{3}-3 g_{2}^{2} M_{2}-\frac{13}{9} g_{1}^{2} M_{1}+h_{b}^{2} A_{b}+6 h_{t}^{2} A_{t}\right)
$$

As one can see, $A_{t}$ receives real contributions $c_{i} M$ proportional to the gaugino mass (whose coefficients $c_{i}$ are different for each sfermion in a generation) and (in principle complex) contributions $d_{i} h_{f}^{2} A_{f}$ from the heavy generation (whose coefficients $d_{i}$ differ for the first two and the third generations); the phases (and magnitudes) of the $A_{i}$ must therefore be run separately. At one loop, the evolution equation for $\mu$ is given by

$$
\frac{d \mu}{d t}=\frac{\mu}{16 \pi^{2}}\left(-3 g_{2}^{2}-g_{1}^{2}+h_{\tau}^{2}+3 h_{b}^{2}+3 h_{t}^{2}\right)
$$

and the phase of $\mu$ does not run.

The aim of this paper is to combine cosmological bounds on the relic density of neutralinos with experimental bounds on the neutron and electron electric dipole moments in order to place limits on the new $C P$-violating phases $\theta_{\mu}$ and $\theta_{A}$. The relic cosmological density of neutralinos and the electric dipole moments are both strongly dependent on the sfermion masses. We take the general form of the sfermion mass ${ }^{2}$ matrix to be [5]

$$
\left(\begin{array}{cc}
M_{L}^{2}+m_{f}^{2}+\cos 2 \beta\left(T_{3 f}-Q_{f} \sin ^{2} \theta_{W}\right) M_{Z}^{2} & m_{f} \bar{m}_{f} e^{i \gamma_{f}} \\
m_{f} \bar{m}_{f} e^{-i \gamma_{f}} & M_{R}^{2}+m_{f}^{2}+\cos 2 \beta Q_{f} \sin ^{2} \theta_{W} M_{Z}^{2}
\end{array}\right)
$$

where $M_{L(R)}$ are the soft supersymmetry breaking sfermion mass which we have assumed are generation independent and generation diagonal and hence real. Due to our choice of phases, there is a non-trivial phase associated with the off-diagonal entries, which we denote by $m_{f}\left(\bar{m}_{f} e^{i \gamma_{f}}\right)$, of the sfermion mass ${ }^{2}$ matrix, and

$$
\bar{m}_{f} e^{i \gamma_{f}}=R_{f} \mu+A_{f}^{*}=R_{f}|\mu| e^{i \theta_{\mu}}+\left|A_{f}\right| e^{-i \theta_{A_{f}}},
$$

where $m_{f}$ is the mass of the fermion $f$ and $R_{f}=\cot \beta(\tan \beta)$ for weak isospin $+1 / 2(-$ $1 / 2$ ) fermions. We also define the sfermion mixing angle $\theta_{f}$ by the unitary matrix $U$ which diagonalizes the sfermion mass ${ }^{2}$ matrix,

$$
U=\left(\begin{array}{cc}
\cos \theta_{f} & \sin \theta_{f} e^{i \gamma_{f}} \\
-\sin \theta_{f} e^{-i \gamma_{f}} & \cos \theta_{f}
\end{array}\right)
$$

Previously [2], it has been shown that the presence of new $C P$-violating phases may have a significant effect on the relic density of bino-type neutralinos. The dominant channel for bino annihilation is into fermion anti-fermion pairs. However, this process exhibits p-wave 
suppression, so that the zero temperature piece of the thermally averaged annihilation crosssection (which is relevant for the annihilation of cold binos) is suppressed by a factor of the final state fermion mass ${ }^{2}$. This significantly reduces the annihilation rate and increases the neutralino relic density. Mixing between left and right sfermions lifts this suppression to some extent by allowing an s-wave contribution to the annihilation cross-section which is proportional to the bino mass ${ }^{2}$, but the presence of complex phases in the off-diagonal components of the sfermion mass matrices dramatically enhances this effect. Explicitly, we compute the relic density by using the method described in ref. [6] and expand $\left\langle\sigma v_{r e l}\right\rangle$ in a Taylor expansion in powers of $T / m_{\widetilde{B}}$

$$
\left\langle\sigma v_{r e l}\right\rangle=a+b\left(T / m_{\widetilde{B}}\right)+O\left(\left(T / m_{\widetilde{B}}\right)^{2}\right)
$$

The coefficients $a$ and $b$ are given by

$$
\begin{gathered}
a=\sum_{f} v_{f} \tilde{a}_{f} \\
b=\sum_{f} v_{f}\left[\tilde{b}_{f}+\left(-3+\frac{3 m_{f}^{2}}{4 v_{f}^{2} m_{\widetilde{B}}^{2}}\right) \tilde{a}_{f}\right]
\end{gathered}
$$

where $\tilde{a}_{f}$ and $\tilde{b}_{f}$ are computed from the expansion of the matrix element squared in powers of $p$, the incoming bino momentum, and $v_{f}=\left(1-m_{f}^{2} / m_{\widetilde{B}}^{2}\right)^{1 / 2}$ is a factor from the phase space integrals. For $M_{L}^{2} \approx M_{R}^{2}$ and $m_{f} \ll m_{\widetilde{B}}$ [2],

$$
\tilde{a}_{f} \approx \frac{\left(g^{\prime}\right)^{4}}{32 \pi} \frac{m_{\widetilde{B}}^{2}}{\left(m_{\tilde{f}}^{2}+m_{\widetilde{B}}^{2}-m_{f}^{2}\right)^{2}} Y_{L}^{2} Y_{R}^{2} \sin ^{2}\left(2 \theta_{f}\right) \sin ^{2} \gamma_{f}+O\left(m_{f} m_{\widetilde{B}}\right)
$$

We see that if sfermion mixing is significant $\left(\theta_{f}\right.$ is large) and the phase of the mixing term is large, the p-wave suppression is removed.

The effect of $C P$-violating phases in a simple model where all the sfermion scalar mass parameters were (for convenience) taken equal at the electroweak scale, along with the trilinear $A$ parameters, was studied in [2]. It was found that the cosmological upper bound on the bino mass in this model was increased from $250 \mathrm{GeV}$ [0, 8] to $650 \mathrm{GeV}$ by the presence of the new phases. This is a significantly stronger effect than the enhancement due to mixing alone [9]. The enhancement found in this case can be partially traced to the assumption of equal scalar masses at the weak scale. In particular, sfermion mixing is sensitive to this assumption as can be seen from the magnitude of $\sin ^{2}\left(2 \theta_{f}\right)$; for $m_{f} \bar{m}_{f} \ll M_{L}^{2}-M_{R}^{2}+2 Q_{f} \cos 2 \beta \sin ^{2} \theta_{W} M_{Z}^{2}$,

$$
\sin ^{2}\left(2 \theta_{f}\right) \approx \frac{m_{f}^{2} \bar{m}_{f}^{2}}{\left(M_{L}^{2}-M_{R}^{2}+2 Q_{f} \cos 2 \beta \sin ^{2} \theta_{W} M_{Z}^{2}\right)^{2}}
$$


The assumption of equal masses translates into taking $M_{L}=M_{R}$ and hence the enhancement in $\sin ^{2}\left(2 \theta_{f}\right)$. Constraints from bounds on the neutron electric dipole moment, however, restrict the range of the relevant phases to $\theta_{\mu}<\pi / 10$ and $\gamma_{\text {down }}<\pi / 6$, so that the $m_{\widetilde{B}}(\max )$ is reduced to about $350 \mathrm{GeV}$. It should be noted however, that the constraints from the neutron electric dipole moment were based on the naive quark model and may in fact be overly restrictive when the strangeness content of the nucleon is taken into account 10.

The above mass ansätz of equal masses at the weak scale is particularly simple. However, this pattern of low-energy sfermion masses contains potentially dangerous charge and colourbreaking minima in the scalar potential [11, 12]. To look at the scalar potential above the electroweak scale, one must RGE evolve the mass $^{2}$ parameters up to the scale of interest. In the MSSM, the sfermion mass ${ }^{2}$ parameters tend to run negative at large scales due to sfermion couplings to gauginos, and for some initial low-energy values of the scalar and gaugino masses, CCB minima appear at high scales. These CCB minima may be avoided by taking the low-energy sfermion masses to be sufficiently high. However, these large sfermion masses lead to a neutralino relic density which is much too large, unless both the common $A$ parameter is tuned so that the lighter of the two stops has a mass close to the neutralino mass and the neutralino is heavier than the top. In contrast, the CMSSM is free of these minima by construction (although other CCB minima may be present [13]). In this case the sfermion mass ${ }^{2}$ parameters are taken non-negative and equal at the unification scale $M_{X}$ and are driven more positive by the gaugino couplings as they are run down to the electroweak scale. In a fully consistent model, one of the Higgs mass ${ }^{2}$ parameters runs negative at low scales to provide $S U(2) \times U(1)$ breaking.

As we have said above, in addition to its simplicity, the CMSSM also enjoys the feature that in most of the parameter space, the lightest neutralino $\chi_{1}^{0}$ (and in fact the LSP) is mostly bino [1], and for large values of the unified gaugino mass $M, \chi_{1}^{0}$ is almost pure bino. We can thus compute the neutralino annihilation rate including only fermion anti-fermion final states (as in (9)), and the error made in not considering higgsino mixing will be insignificant for the relevant gaugino masses. In Figure 1, we show contours of constant neutralino relic density, in the $M-m_{0}$ plane. Throughout this paper we use $\tan \beta=2.1$ and $m_{\text {top }}=170 \mathrm{GeV}$. Three contours are shown, for $\Omega_{\tilde{\chi}} h^{2}$ of $0.25,0.5$, and 1 . The two vertical contours are bino purity contours of 0.95 and 0.99 ; we see that for bino masses near their upper bounds for $\Omega_{\widetilde{\chi}} h^{2}<0.25$, where $m_{\widetilde{B}} \approx 0.4 M \approx 160 \mathrm{GeV}$, the higgsino admixture in the LSP is extremely small. The shaded regions in Figure 1 are ruled out because they lead either to a chargino with a mass less than $\sim 65 \mathrm{GeV}$ [14], a sfermion with a mass less than $74 \mathrm{GeV}$ [15], or a stop, chargino, or (in the bottom right region) stau as the LSP. The contours of $\Omega_{\tilde{\chi}} h^{2}$ 
were computed for the particular choices of $A_{0}=300 \mathrm{GeV}, \theta_{\mu}=0, \theta_{A}=0.8 \pi$; however, the contours vary little as these parameters are changed, and the variation is already completely negligible at the upper range of the allowed bino mass for $\Omega_{\tilde{\chi}} h^{2}=0.25$. There is a similar picture for $\theta_{\mu}=\pi(\mu \rightarrow-\mu)$.

In contrast to the model studied in [2], the upper bound on the bino mass in the CMSSM is essentially independent of the $C P$-violating phases $\theta_{\mu}$ and $\theta_{A}$. Recall from (9) that the phases are only important if there is a significant amount of L-R sfermion mixing. In the CMSSM, when $M_{L}^{2}$ and $M_{R}^{2}$ are run down from $M_{X}$, they are split by roughly $0.4 M^{2}$ at the electroweak scale. From an examination of Eq. (10), one can see that the off-diagonal components of the sfermion mass matrices which contain powers of the fermion mass are much less than $M_{L}^{2}-M_{R}^{2}$ when $M$ is a few hundred $\mathrm{GeV}$, therefore sfermion mixing is negligible, and the phases cannot lift the p-wave suppression. Additionally, since at the upper range of bino masses we are deep in the pure bino region, the composition of the lightest neutralino is independent of $\mu$, and $\theta_{\mu}$ in particular. One can therefore set an upper bound for $M$ from cosmological considerations which is quite independent of parameter choices. (If one takes $\tan \beta$ very large so that stau mixing is significant, then the neutron and electron electric dipole moments given below become very large as well. Constraints on the $C P$-violating phases from bounds on EDM's then ensure that effect of the phases on the neutralino relic density are negligible.) One finds that for $\Omega_{\tilde{\chi}} h^{2}<0.25, M$ must be less than about $400 \mathrm{GeV}$, which corresponds to a bino mass of roughly $160 \mathrm{GeV}$.

We turn now to the calculation of the electric dipole moments of the neutron and the electron. As our calculation of the EDM's parallels our previous calculation [2], we refer the reader there for details. As in [2], we will only include the three contributions coming from neutralino, chargino, and gluino exchange to the quark electric dipole moment. The necessary $C P$ violation in these contributions comes from either $\gamma_{f}$ in the sfermion mass matrices or $\theta_{\mu}$ in the neutralino and chargino mass matrices. Full expressions for the chargino, neutralino and gluino exchange contributions are found in [16].

The contributions to the quark electric dipole moments from the individual gaugino exchange diagrams fall as $M$ is increased, because the squark masses ${ }^{2}$ receive large contributions proportional to $M^{2}$ during their RGE evolution from $M_{X}$ to $M_{Z}$. Roughly,

$$
m_{\widetilde{q}}^{2} \approx m_{0}^{2}+6 M^{2}+O\left(M_{Z}^{2}\right)
$$

Thus even for large values of the $C P$ violating phases, one can always turn off the quark electric dipole moment contributions to the neutron EDM by making $M$ sufficiently large [16]; however one must still satisfy the cosmological bounds discussed above. Experimental bounds 
are $\left|d_{n}\right|<1.1 \times 10^{-25} e \mathrm{~cm}$ [17] for the neutron electric dipole moment and $\left|d_{e}\right|<1.9 \times$ $10^{-26} e \mathrm{~cm}[18]$ for the electron EDM. Note also that the squark masses $m_{\widetilde{q}}^{2}$ are only weakly dependent on $m_{0}$ in the cosmologically allowed regions of Figure 1, and so the quark EDM's will also be independent of $m_{0}$.

We have computed the neutron EDM in the CMSSM as a function of $\theta_{\mu}, \theta_{A}$, and $M$ for fixed $A_{0}, m_{0}$, and $\tan \beta$, using the naïve quark model. In practice we find that the dominant contribution to the quark electric dipole moments in the CMSSM come from the chargino exchange diagrams, unless $\theta_{\mu}$ is extremely small (and $\theta_{A} \gg \theta_{\mu}$ ). We can then find, as a function of $\theta_{\mu}$ and $\theta_{A}$, the minimum value of $M$ required to bring the quark electric dipole moment contributions to the neutron EDM below the experimental limits. In Figure 2, we plot contours of $M_{\min }$ for $A_{0}=300 \mathrm{GeV}$ and $m_{0}=100 \mathrm{GeV}$. The light central region corresponds to $M_{\min }<200 \mathrm{GeV}$, and successive contours represent steps of $100 \mathrm{GeV}$. The darkest regions on the left and right sides of the figure lead to a stau as the LSP and correspond to the lower right shaded region in Figure 1; their positions do depend somewhat on $m_{0}$, as can be seen from Figure 1. The EDM's were computed on a grid with spacings of $\pi / 10$ in $\theta_{A}$ and $\pi / 50$ in $\theta_{\mu}$, so contour features smaller than these dimensions are not significant. Recalling from Figure 1 that $\Omega_{\tilde{\chi}} h^{2}<0.25$ requires $M<400 \mathrm{GeV}$, we find the constraint that $\left|\theta_{\mu}\right| \lesssim \pi / 15$ (the region with $\theta_{\mu}<0$ comes from values of $\theta_{A}$ near $3 \pi / 2$ ). There is a similar allowed region near $\theta_{\mu}=\pi$, corresponding to a sign change in $\mu$. The dependence on $\theta_{A}$ in Figure 2 is weak because it affects only the gluino exchange contribution, which is sub dominant, and even then $\theta_{A}$ is only partially responsible for the relevant phases $\gamma_{\text {up }}$ and $\gamma_{\text {down }}$ (see Eq. (四)). The figure is shifted slightly to one side of $\theta_{\mu}=0$ because in one direction there is a cancellation between the chargino and gluino exchange pieces, and in the other direction the two contributions come in with like signs. These bounds are not dependent on $m_{0}$ and are only very weakly dependent on $A_{0}$ for $A_{0}<1 \mathrm{TeV}$.

In ref [2] it was found that parameter choices which produced a sufficiently small neutron EDM also produced an electron EDM well below experimental bounds, so that it was never necessary to separately consider bounds from the electron EDM. In the case of the CMSSM, this is not always the case. The reason is that in the CMSSM, the squarks receive a large contribution to their masses from the RGE running and are consequently much heavier than the sleptons, in contrast to the case studied in ref [2] where all the sfermions shared a common scalar mass $^{2}$ parameter at $M_{Z}$. The analysis for the electron EDM is slightly complicated by the fact that the slepton masses, and consequently the electron EDM, do depend somewhat on $m_{0}$ in the cosmologically allowed region, even at the upper limit of the neutralino mass, where $M$ takes its largest allowed value. However, for values of $m_{0}$ below $100 \mathrm{GeV}$, this 
dependence is not strong, and the minimum values of $M$ we find change by less than $10 \%$ for values of $m_{0}<100 \mathrm{GeV}$. In Figure 3 we show contours of constant $M_{\text {min }}$ for $A_{0}=300 \mathrm{GeV}$ and $m_{0}=100 \mathrm{GeV}$, with the requirement that $\left|d_{e}\right|<1.9 \times 10^{-26} e \mathrm{~cm}$ 18. Again, the darkest regions on the left and right sides of the plot lead to a stau as the LSP and correspond to the lower right shaded region in Figure 1. This time the slight dependence on $\theta_{A}$ comes from the dependence of the neutralino exchange contribution (which in this case is significant) on the phase $\theta_{A}$. We observe that we get comparable bounds to those from the neutron EDM in Figure $2,\left|\theta_{\mu}\right| \lesssim \pi / 10$. It is important to note however, that the bounds coming from the electron electric dipole moment are clearly not sensitive to the spin structure of the nucleon 10 .

In summary, we have combined cosmological bounds on gaugino masses with experimental bounds on the neutron and electron electric dipole moments to constrain the new $C P$-violating phases in the Constrained Minimal Supersymmetric Standard Model. We find that in contrast to models studied previously, the phases do not affect the cosmological limits on the mass of an LSP bino-type neutralino. While there is no bound on the phase $\theta_{A}$ of the unified trilinear scalar mass parameter $A$, the phase of the supersymmetric Higgs mixing mass is constrained by $\left|\theta_{\mu}\right| \lesssim \pi / 10$.

\section{Acknowledgments}

We would like to thank Mark Srednicki for continual discussions. This work was supported in part by DOE grant DE-FG02-94ER-40823.

\section{References}

[1] J.Ellis, J. Hagelin, and D.V. Nanopoulos, Phys. Lett. B159 (1985) 26; M.M. Nojiri, Phys. Lett. B261 (1991) 76; J. Lopez, D.V. Nanopoulos, and K. Yuan, Phys. Lett. B267 (1991) 219; and Nucl. Phys. B370 (1992) 445; J. Ellis, and L. Roszkowski, Phys. Lett. B283 (1992) 252; M. Drees and M.M. Nojiri, Phys. Rev. D47 (1993) 376; S. Kelly, J.L. Lopez, D.V. Nanopoulos, H. Pois, and K. Yuan, Phys. Rev. 47 (1993) 2461; S.A. Abel, S. Sarkar, and I.B. Whittingham, Nucl. Phys. B392 (1993) 83; R.G. Roberts and L. Roszkowski, Phys. Lett. B309 (1993) 329; R. Arnowitt, and P. Nath, Phys. Rev. 
Lett. 70 (1993) 3696; G. Kane, C. Kolda, L. Roszkowski, J. Wells, Phys. Rev. D49 (1994) 6173.

[2] T. Falk, K.A. Olive and M. Srednicki, Phys. Lett. 354 (1995) 99.

[3] M. Dugan, B. Grinstein and L. Hall, Nucl. Phys. B255, 413 (1985).

[4] K. Inoue, A. Kakuto, H. Komatsu and S. Takeshita, Prog. Th. Phys. 68 (1982) 927; M. Drees and M. Nojiri, Phys. Rev. D47 (1993) 376; W. de Boer, R. Ehret, W. Oberschulte, and D.I. Kazakov, Z. Phys. C67 (1995) 647; S.P. Martin and M.T. Vaughn, Phys. Rev. D50 (1994) 2282.

[5] J. Ellis and S. Rudaz, Phys. Lett. B128 (1983) 248.

[6] M. Srednicki, R. Watkins and K.A. Olive, Nucl. Phys B310 (1988) 693.

[7] K.A. Olive and M. Srednicki, Phys. Lett. B230 (1989) 78; Nucl. Phys. B355 (1991) 208.

[8] K. Greist, M. Kamionkowski, and M.S. Turner, Phys. Rev. D41 (1990) 3565.

[9] T. Falk, R. Madden, K.A. Olive, and M. Srednicki, Phys. Lett. B318 (1993) 354.

[10] J. Ellis and R. Flores, hep-ph/9602211 (1996).

[11] T. Falk, K.A. Olive, M. Srednicki and L. Roszkowski, UMN-TH-1411-95, hepph/9510308.

[12] A. Riotto and E. Roulet, hep-ph/9512401 (1995).

[13] C.Kounnas, A.B. Lahanas, D.V. Nanopoulos and M. Quirós, Nucl. Phys. B236 (1984) 438; J.M. Frère, D.R.T. Jones and S. Raby, Nucl. Phys. B222 (1983) 11; J.F. Gunion, H.E. Haber and M. Sher, Nucl. Phys. B306 (1988) 1; J.A. Casas, A. Lleyda and C. Muñoz, FTUAM95/11, hep-ph/9507294.

[14] The ALEPH Collaboration, CERN-PPE/96/10 (1996).

[15] J. Alitti et al., Phys. Lett. B235 (1990) 363.

[16] Y. Kizukuri \& N. Oshimo, Phys. Rev. D45 (1992) 1806; D46 (1992) 3025.

[17] I. S. Altarev et al., Phys. Lett B276 (1992) 242.

[18] K. Abdullah et al., Phys. Rev. Lett. 65, 2347 (1990). 


\section{Figure Captions}

Fig. 1) Contours of constant $\Omega_{\tilde{\chi}} h^{2}=0.25,0.5$, and 1.0, as a function of $m_{0}$ and $M$, for $A_{0}=300 \mathrm{GeV}, \theta_{A}=0.8 \pi, \theta_{\mu}=0$, and $\tan \beta=2.1$. The vertical lines represent contours of constant bino purity, $p=0.95$ and $p=0.99$. The shaded regions yield an LSP which is either a chargino or a sfermion.

Fig. 2) Contours of constant $M_{\text {min }}$, the minimum gaugino mass parameter needed to bring the neutron electric dipole moment below experimental bounds, for $A_{0}=300 \mathrm{GeV}, m_{0}=100 \mathrm{GeV}$. The light central region corresponds to $M_{\min }<$ $200 \mathrm{GeV}$, and successive contours represent steps of $100 \mathrm{GeV}$. Note that $M<$ $400 \mathrm{GeV}$ implies $\theta_{\mu} \lesssim \pi / 15$.

Fig. 3) Same as Fig. 2 for the electron electric dipole moment. In this case $M<$ $400 \mathrm{GeV}$ implies $\theta_{\mu} \lesssim \pi / 10$. 
Figure 1

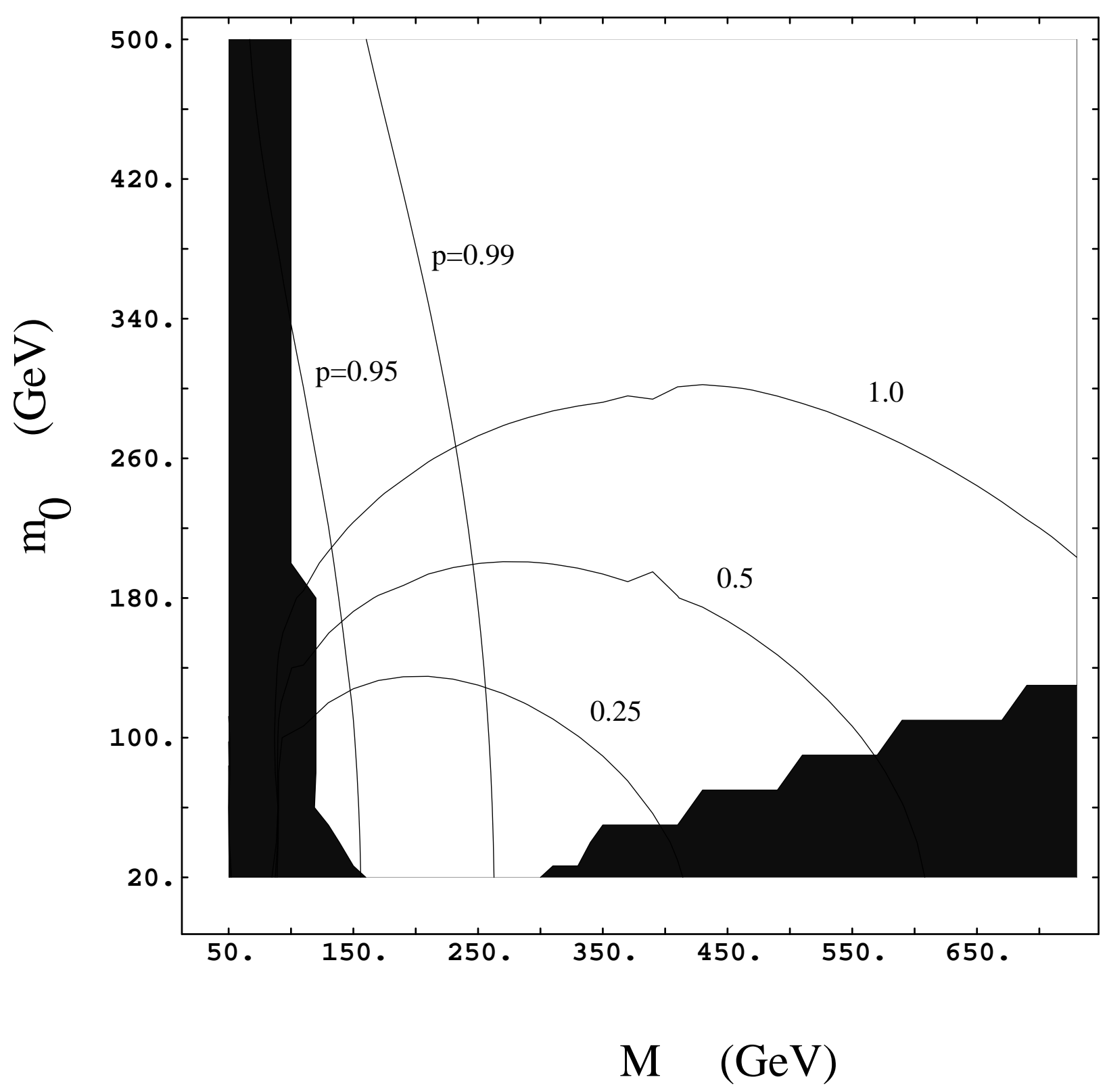


Figure 2

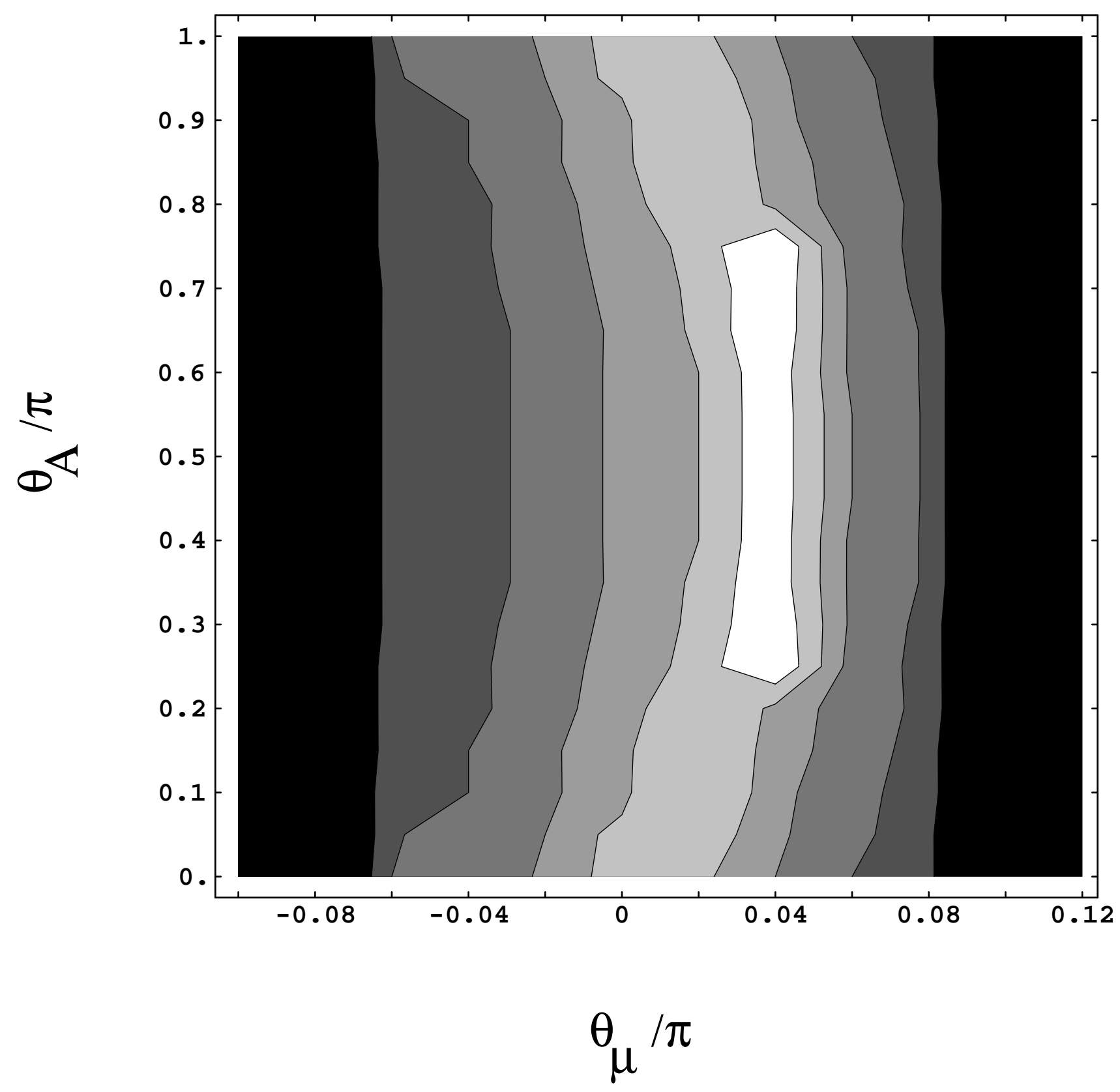


Figure 3

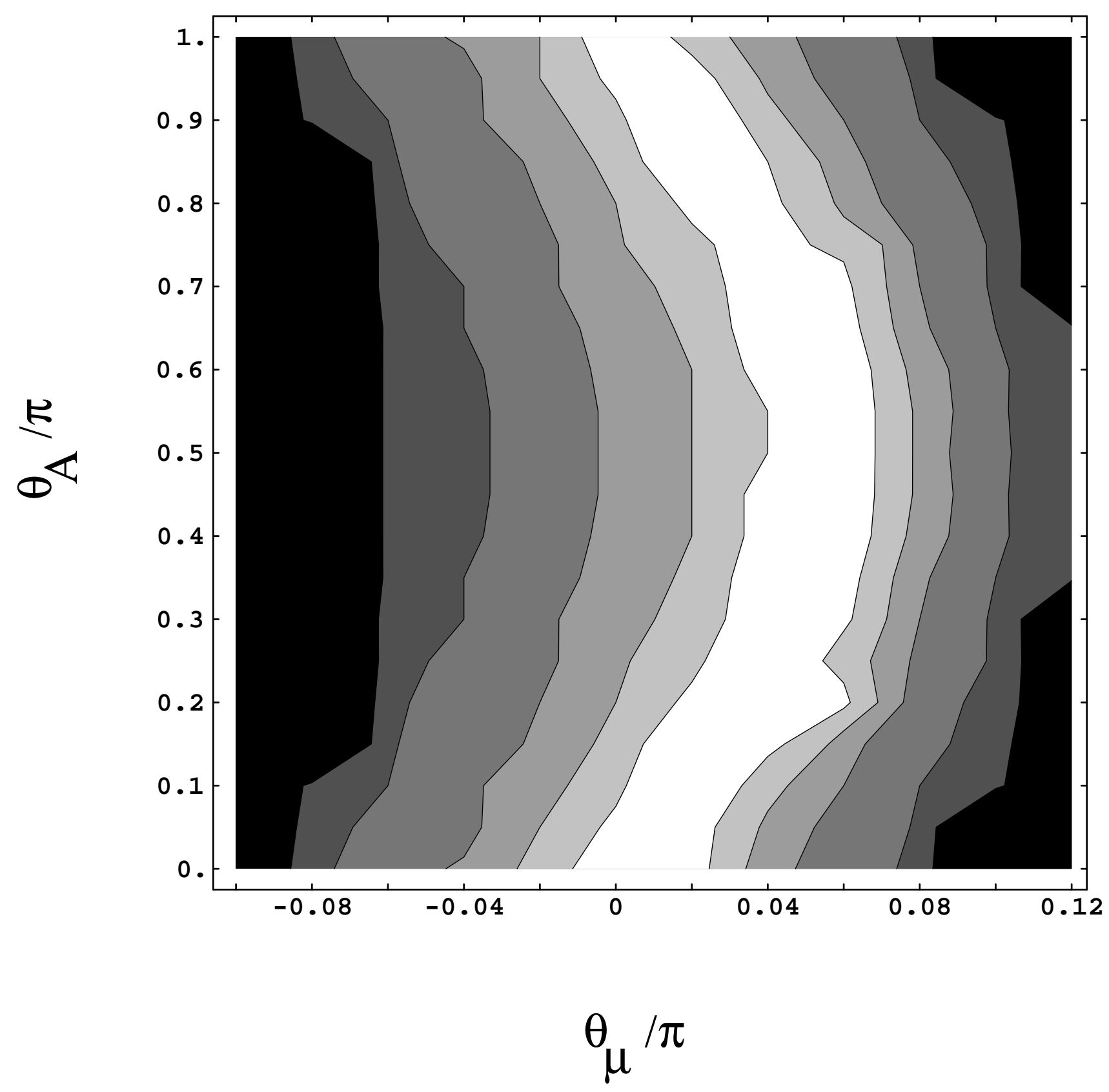

